\title{
Discretization of Volterra Integral Equations of the First Kind
}

\author{
By Hermann Brunner
}

\begin{abstract}
We show that various (discrete) methods for the approximate solution of Volterra (and Abel) integral equations of the first kind correspond to some discrete version of the method of (recursive) collocation in the space of (continuous) piecewise polynomials. In a collocation method no distinction has to be made between equations with regular or weakly singular kernels; the regularity or nonregularity of the given integral operator becomes only relevant when selecting a discretization procedure for the moment integrals resulting from collocation. Similar results hold for equations of the second kind.
\end{abstract}

1. Introduction. Let the subspace $C_{a}(I) \subset C(I)$ be defined by $C_{a}(I)=\{f \in$ $C(I): f(a)=0$, where $I=[a, b]$ is a compact interval $(a<b)$, and denote by $T$ : $C(I) \rightarrow C_{a}(I)$ the Volterra integral operator

$$
(T f)(x)=\int_{a}^{x} K(x, t) f(t) d t, \quad x \in I .
$$

Here, the kernel $K$ of $T$ is supposed to be such that either

$$
K \in C(S) \quad \text { (where } S=\{(x, t): a \leqslant t \leqslant x \leqslant b\}),
$$

or

$$
K(x, t)=G(x, t)(x-t)^{-\lambda}, \quad \lambda \in(0,1), \text { with } G \in C(S) .
$$

We shall require one additional hypothesis (compare also Section 2): the operator $T$ will be called strictly monotone (or: positive [5]) if its kernel $K$ also satisfies either

$$
|K(x, t)| \geqslant r_{0}>0, \quad(x, t) \in S \text { (for the case (1.2a)), }
$$

or

$$
|G(x, t)| \geqslant r_{0}>0, \quad(x, t) \in S \text { (for the case (1.2b)). }
$$

For a given function $g \in C_{a}(I)$ the Volterra integral equation of the first kind,

$$
(T y)(x)=g(x), \quad x \in I,
$$

is to be solved in the space $C(I)$. (Here, the expression "Volterra integral equation" is meant to include both the classical Volterra equation correspondingoto (1.2a) and the (generalized) Abel equation with kernel (1.2b).)

Received February 17, 1976; revised August 20, 1976.

AMS (MOS) subject classifications (1970). Primary 65R05; Secondary 65D05, 65D30.

Key words and phrases. First-kind integral equations of Volterra and Abel type, collocation by piecewise polynomials, numerical quadrature, discrete appoximations. 
Among the methods for the approximate solution of (1.4) suggested in recent years, we mention, in the context of the following discussion, one-step methods based on finite-difference techniques (compare Linz [10]), those methods using the idea of product integration introduced by Young [16] (see Linz [12], Weiss [14], Weiss and Anderssen [15]), and the higher-order block methods of de Hoog and Weiss [6] , [7] (for a brief summary, compare also [1]). It is the purpose of this note to show that all these methods (in which the cases (1.2a) (regular kernel) and (1.2b) (weakly singular kernel) had to be treated separately) may be regarded as particular discretizations of methods based on the idea of (recursive) collocation ([3], [4]), where the exact solution $y$ of (1.4) is projected into the space of piecewise polynomials $s(x)$ of degree $m$ and with appropriate knots $Z_{N} \subset I: s(x)$ is either of continuity class $C(I)$ (see Section 2), or $s(x)$ possesses discontinuities on $Z_{N}$ (Section 3). If a given Volterra equation (1.4) is solved by recursive collocation, it is clear that no distinction has to be made (in contrast to the various discrete methods just mentioned) between regular (classical Volterra) operators and weakly singular (generalized Abel) operators; furthermore, methods using recursive collocation allow for an important degree of flexibility in that they can be used for highly oscillatory (fast varying) kernels without increasing $m$ or the number of knots $Z_{N} \subset I$ simply by selecting an appropriate discretization procedure when evaluating the resulting moment integrals.

2. Recursive Collocation in $S_{m}^{(0)}\left(Z_{N}\right)$. Let $\Delta_{N}: a=x_{0}<x_{1}<\cdots<x_{N}=$ $b(N \geqslant 1)$ denote a partition of $I$, and define $Z_{N}=\left\{x=x_{k}: k=1, \ldots, N-1\right\}, \sigma_{k}$ $=\left[x_{k}, x_{k+1}\right](k=0,1, \ldots, N-1)$. Since the discrete methods for solving (1.4) mentioned in Section 1 are usually applied with a uniform stepsize we shall deal, in order to simplify the notation, only with special partitions $\Delta_{N}$ where $x_{k}=a+k h, k$ $=0,1, \ldots, N$ (although all the results hold for arbitrary $\Delta_{N}$ ).

We define the subspace $S_{m}^{(0)}\left(Z_{N}\right)$ of $C(I)$ by

$$
S_{m}^{(0)}\left(Z_{N}\right)=\left\{\Phi \in C(I):\left.\Phi\right|_{\sigma_{k}}=\Phi_{k} \in \pi_{m}, k=0,1, \ldots, N-1\right\} \quad(m \geqslant 1),
$$

i.e. $S_{m}^{(0)}\left(Z_{N}\right)$ is the space of (continuous) piecewise polynomials of degree $m \geqslant 1$ and with knots $Z_{N}$. For $x \in \sigma_{k}$ we set

$$
\Phi_{k}=\Phi_{k}\left(\alpha_{k}, x\right)=\sum_{\nu=0}^{m} \alpha_{k, \nu} \phi_{k, \nu}(x),
$$

where $\left\{\phi_{k, \nu}\right\}$ denotes a given basis in $\pi_{m}$ (on $\sigma_{k}$ ), and where

$$
\alpha_{k}=\left(\alpha_{k, 0}, \alpha_{k, 1}, \ldots, \alpha_{k, m}\right)^{T} \in \mathbf{R}^{m+1} \quad(k=0,1, \ldots, N-1) .
$$

For brevity we shall often write $\Phi_{k}(x)$ for $\Phi_{k}\left(\alpha_{k}, x\right)$.

The given integral equation (1.4) is now solved in the space $S_{m}^{(0)}\left(Z_{N}\right)$ (rather than in $C(I)$ ) by requiring that, for $k=0,1, \ldots, N-1$,

$$
(T \Phi)(x)=g(x), \quad x \in X_{k},
$$

where $X_{k} \subset \sigma_{k}$ is the set 


$$
X_{k}=\left\{x=\xi_{k, j}=x_{k}+\eta_{j} h: 0<\eta_{1}<\cdots<\eta_{m} \leqslant 1\right\} .
$$

The collocation condition (2.2) may be stated in a form which exhibits more clearly the recursive generation of the approximating function $\Phi \in S_{m}^{(0)}\left(Z_{N}\right)$, namely

$$
\int_{x_{k}}^{x} K(x, t) \Phi_{k}(t) d t=g(x)-\sum_{\mu=0}^{k-1} \int_{x_{\mu}}^{x_{\mu+1}} K(x, t) \Phi_{\mu}(t) d t,
$$

$$
x \in X_{k}(k=0,1, \ldots, N-1)
$$

in addition, the continuity conditions for $Z_{N}$ yield

$$
\Phi_{k}\left(x_{k}\right)=\Phi_{k-1}\left(x_{k}\right), \quad x_{k} \in Z_{N} .
$$

For $x=x_{0}=a$ we set

$$
\Phi_{0}\left(x_{0}\right)=y(a) .
$$

(We note here that, in order to avoid the evaluation of the value of the unknown exact solution at $x=a$, the representation of $\Phi$ on the initial interval $\sigma_{0}$ may be computed by using collocation on a suitable set $\hat{X}_{0} \subset\left(x_{0}, x_{1}\right]$ consisting of $(m+1)$ distinct points. This will not be pursued further since, as was pointed out before, our interest lies in the relationship between collocation methods and discrete methods which, in general, have to rely on the starting value $y(a)$. But compare also Section 3 .)

We now rewrite (2.4) in the form

$$
C_{k} \alpha_{k}=r_{k}, \quad k=0,1, \ldots, N-1,
$$

where the components $\left\{r_{k, j}: j=1, \ldots, m\right\}$ of $r_{k} \in \mathbf{R}^{m+1}$ are given by the righthand side of (2.4a) corresponding to $x=\xi_{k, j} \in X_{k}$; its first component $r_{k, 0}$ is obtained by observing (2.4b), (2.4c). The matrix $C_{k}=\left(c_{j, \nu}^{(k)}\right)_{m+1}$ in (2.5) possesses the elements

$$
c_{j, \nu}^{(k)}=\int_{x_{k}}^{\xi_{k, j}} K\left(\xi_{k, j}, t\right) \phi_{k, \nu}(t) d t \quad(j=1, \ldots, m ; \nu=0,1, \ldots, m),
$$

and (by (2.4b))

$$
c_{0, \nu}^{(k)}=\phi_{k, \nu}\left(x_{k}\right), \quad \nu=0,1, \ldots, m .
$$

If the operator $T$ in (1.4) is strictly monotone (i.e. if $T$ satisfies one of the corresponding pairs of conditions in (1.2) and (1.3)), then $C_{k}$ is nonsingular for any set $X_{k}$ and any $h>0(k=0,1, \ldots, N-1)$, since any basis $\left\{\phi_{k, \nu}\right\}$ for $\pi_{m}$ constitutes a Chebyshev system on $\sigma_{k}$ [5]. Hence, for such operators $T$, (2.4) (or (2.5)) defines a unique projection of the exact solution $y$ of (1.4) in the subspace $S_{m}^{(0)}\left(Z_{N}\right)$. On the other hand, any of the methods described in [10], [14], [15], [7] yields a projection of $y$ in the space $\mathbf{R}^{p}$, with $p=m N+1$.

In order to establish the link between these two classes of methods we now select the following bases $\left\{\phi_{k, \nu}\right\}$. Let the sets $\left\{Q_{k}\right\}$ be given by 


$$
\begin{aligned}
Q_{k}=\left\{x=q_{k, j}=x_{k}+u_{j} h: 0 \leqslant u_{0}<u_{1}<\right. & \left.\cdots<u_{m} \leqslant 1\right\} \\
& (k=0,1, \ldots, N-1) .
\end{aligned}
$$

For a fixed $Q_{k}$ let $\left\{l_{k, \nu}(x): \nu=0,1, \ldots, m\right\}$ denote the corresponding Lagrange fundamental polynomials; as the basis in $\pi_{m}$ we then choose $\left\{\phi_{k, \nu}(x)\right\}=\left\{l_{k, \nu}(x)\right\}$. Relation (2.1) yields immediately

$$
\Phi_{k, j}=\Phi_{k}\left(q_{k, j}\right)=\alpha_{k, j}, \quad j=0,1, \ldots, m,
$$

since $l_{k, \nu}\left(q_{k, j}\right)=\delta_{\nu, j}$.

The following result is now obvious.

THEOREM 1. Assume:

(i) The operator $T$ in (1.4) is strictly monotone.

(ii) For a fixed $k, 0 \leqslant k \leqslant N-1$, the (unique) approximation $\Phi \in S_{m}^{(0)}\left(Z_{N}\right)$ to $y$ on $\left[x_{0}, x_{k+1}\right]$ has been generated by (2.4) (collocation on the sets $X_{\mu}: \mu=0$, $1, \ldots, k)$.

Then the values $\left\{\Phi_{k, \nu}\right\}$ of $\Phi$ on $Q_{k}$ are given by

$$
\begin{aligned}
\int_{x_{k}}^{\xi_{k, j}} K\left(\xi_{k, j}, t\right) \sum_{\nu=0}^{m} \Phi_{k, \nu} l_{k, \nu}(t) d t & \\
& =g\left(\xi_{k, j}\right)-\sum_{\mu=0}^{k-1} \int_{x_{\mu}}^{x_{\mu+1}} K\left(\xi_{k, j}, t\right) \Phi_{\mu}(t) d t, \quad j=1, \ldots, m,
\end{aligned}
$$

with $\Sigma_{\nu=0}^{m} \Phi_{k, \nu} l_{k, \nu}\left(x_{k}\right)=\Phi_{k-1}\left(x_{k}\right)$.

It has already been briefly indicated that Theorem 1 holds independent of whether the kernel $K$ of $T$ is of the form (1.2a) (regular) or (1.2b) (weakly singular). In general, however, it will not be possible to evaluate the moment integrals occurring in (2.9) explicitly: some appropriate discretization procedure has to be chosen to approximate them. It is only here that a distinction between regular and weakly singular kernels has to be made (note that the integrals over the intervals $\left[x_{\mu}, x_{\mu+1}\right]$, $\mu=0,1, \ldots, k-1$, occurring in $r_{k}$ are, in the case (1.2b), no longer singular since $\left.\xi_{k, j}>x_{k}\right)$. The discretization procedure one selects will depend on $m$ as well as on the behavior of $K$ (or $G$, respectively), and one has, among others, the following options:

(i) Discretization is based on one of the many available automatic quadrature routines which compute the integrals in (2.6a) to a specified accuracy (compare, for example, the recent report [13], as well as related work by Lyness and Kaganove). Such an approach will be especially appropriate if the kernel of $T$ is a rapidly varying function; here, the classical finite-difference methods [10] and the methods based on product integration [14], [15] (which, as will be shown below, correspond to rather crude discretization procedures for (2.9)) will not be feasible unless $h$ is small.

(ii) For $K \in C(S)$ (case (1.2a)), and for a fixed value of $j(1 \leqslant j \leqslant m)$ in 
(2.9), let the integrals (2.6) be evaluated by an interpolatory quadrature formula (on $\left.\left[x_{k}, \xi_{k, j}\right]\right)$, defined by weights $\left\{w_{j, r}: r=0,1, \ldots, m\right\}$ and abscissas $\left\{z_{j, r}^{(k)}\right\}$, where

$$
z_{j, r}^{(k)}=x_{k}+\eta_{j} \eta_{r} h=x_{k}+\eta_{r}\left(\xi_{k, j}-x_{k}\right)
$$

$r=0,1, \ldots, m$ (with the quantities $\left\{\eta_{j}\right\}$ as defined in (2.3), $\eta_{0}=0$ : affine transformation of the points $X_{k}$ ). The integrals occurring in the $j$ th component of $r_{k}$ in (2.9) are discretized accordingly (by using the weights $\left\{w_{m, r}\right\}$, with $w_{j, r}=\eta_{j} w_{m, r}(j$ $=1, \ldots, m)$, and the abscissas $\left\{\xi_{\mu, r}\right\}$ on the intervals $\left[x_{\mu}, x_{\mu+1}\right], \mu=0,1, \ldots$, $k-1)$. The discretized form of the left-hand side of (2.9) thus reads

$$
\sum_{r=0}^{m} w_{j, r} K\left(\xi_{k, j}, x_{k}+\eta_{j} \eta_{r} h\right) \sum_{\nu=0}^{m} \Phi_{k, \nu} l_{k, \nu}\left(x_{k}+\eta_{j} \eta_{r} h\right)
$$

If we choose in particular $\eta_{j}=u_{j}, j=0,1, \ldots, m$, with $u_{0}=0, u_{m}=1$, then the resulting discretization procedure for (2.9) yields the block method suggested by de Hoog and Weiss [7] (method (3.3)); here, (2.10) reduces to

$$
\sum_{r=0}^{m} w_{j, r} K\left(q_{k, j}, x_{k}+u_{j} u_{r} h\right) \sum_{\nu=0}^{m} \Phi_{k, \nu} l_{k, \nu}\left(x_{k}+u_{j} u_{r} h\right) \quad(j=1, \ldots, m),
$$

whereas the $j$ th component of $r_{k}$ is replaced by

$$
g\left(q_{k, j}\right)-\sum_{\mu=0}^{k-1} \sum_{r=0}^{m} w_{m, r} K\left(q_{k, j}, q_{\mu, r}\right) \Phi_{\mu, r} \quad\left(\Phi_{0,0}=y(a)\right) .
$$

(iii) If the kernel of $T$ is of the form (1.2b) (weakly singular), then (2.9) can be discretized in an analogous fashion: we now require that $u_{m}<1$ since the singularity occurs, for a fixed value of $j$, at $t=\xi_{k, j}$. In particular, Gauss-type quadrature formulas will then be a suitable choice, (We mention here that, for nonsmooth kernels, discretization procedures based on product-type quadrature introduced by Boland and Duris [2] will often prove to be suitable alternatives, both for regular and weakly singular kernels.)

To conclude this section we briefly consider the special case $m=1$. Here, it will be convenient to choose the basis functions $\phi_{k, \nu}(x)=\left[\left(x-x_{k}\right) / h\right]^{\nu}, x \in \sigma_{k}(\nu=$ $0,1)$.

THEOREM 2. Let the kernel $K$ of $T$ satisfy the conditions stated in Theorem 1. Then, for $m=1$, the approximating function $\Phi \in S_{1}^{(0)}\left(Z_{N}\right)$ to the exact solution of (1.4) is uniquely determined by (2.4) and is given by

$$
\alpha_{k, 1}=\left\{\int_{x_{k}}^{\xi_{k, 1}} K\left(\xi_{k, 1}, t\right) \phi_{k, 1}(t) d t\right\}^{-1}
$$

$$
\begin{array}{r}
\left\{g\left(\xi_{k, 1}\right)-\sum_{\mu=0}^{k-1} \int_{x_{\mu}}^{x_{\mu+1}} K\left(\xi_{k, 1}, t\right) \Phi_{\mu}(t) d t-\alpha_{k, 0} \int_{x_{k}}^{\xi_{k, 1}} K\left(\xi_{k, 1}, t\right) d t\right\} \\
(k=0,1, \ldots, N-1)
\end{array}
$$

with $\alpha_{0,0}=y(a)$, and with $\alpha_{k, 0}$ given by (2.4b). 
The proof is straightforward (compare (2.4a) and (2.6a)) and is hence omitted.

Again, a number of well-known methods for the numerical solution of (1.4) may be obtained from (2.11) by specifying the type of kernel of $T$ and the discretization procedure for the moment integrals. We mention two examples:

(i) If $K \in C(S)$, and if the trapezoidal rule is used (with stepsize $h$, and by choosing $\xi_{k, 1}=x_{k+1}$ ), then (2.11) clearly yields the trapezoidal method [10].

(ii) Let now $K$ be of the form (1.2b) (weakly singular case). Then, if the integrals in (2.11) are regarded as weighted integrals, with the weight functions

$$
w_{\mu}(t)=\left(\xi_{k, 1}-t\right)^{-\lambda} \quad(\mu=0,1, \ldots, k),
$$

discretization by the (weighted) trapezoidal rule (stepsize $h, \xi_{k, 1}=x_{k+1}$ ) furnishes the product integration method suggested by Weiss [14]

3. Recursive Collocation in $S_{m-1}^{(-1)}\left(Z_{N}\right)$. In the present section we shall show that certain discrete methods for solving (1.4) correspond to special discretizations of recursive collocation methods in the space of piecewise polynomials which possess discontinuities (finite jumps) on $Z_{N}$. As before, let $m \geqslant 1$, and let $\Delta_{N}$ and $Z_{N}$ be as in Section 2, with $\sigma_{k}^{\prime}=\left(x_{k}, x_{k+1}\right](k=0,1, \ldots, N-1)$. We define the space $S_{m-1}^{(-1)}\left(Z_{N}\right)$ by

$$
S_{m-1}^{(-1)}\left(Z_{N}\right)=\left\{\Phi:\left.\Phi\right|_{\sigma_{k}^{\prime}} \in \pi_{m-1}, k=0,1, \ldots, N-1\right\}
$$

i.e. $S_{m-1}^{(-1)}\left(Z_{N}\right)$ consists of piecewise polynomials of degree $(m-1)$ (with knots $Z_{N}$ ) which have (finite) discontinuities on $Z_{N}$. For $x \in \sigma_{k}^{\prime}$ we set for $\Phi \in S_{m-1}^{(-1)}\left(Z_{N}\right)$

$$
\Phi_{k}=\Phi_{k}\left(\alpha_{k}, x\right)=\sum_{\nu=1}^{m} \alpha_{k, \nu} \phi_{k, \nu}(x),
$$

where now $\left\{\phi_{k, \nu}\right\}$ denotes some basis for $\pi_{m-1}$ (on $\sigma_{k}^{\prime}$ ), and where $\alpha_{k} \in \mathbf{R}^{m}$. Furthermore, let $X_{k}$ be as in (2.3), and define the sets $Q_{k}^{\prime} \subset \sigma_{k}^{\prime}$ by

$$
Q_{k}^{\prime}=\left\{x=q_{k, j}^{\prime}=x_{k}+u_{j} h: 0<u_{1}<\cdots<u_{m} \leqslant 1\right\} .
$$

In analogy with the procedure described in Section 2, an element $\Phi \in S_{m-1}^{(-1)}\left(Z_{N}\right)$ approximating the exact solution $y$ of (1.4) on $I$ will be generated recursively by collocation on the sets $\left\{X_{k}\right\}$, i.e. we have again the collocation conditions (2.4a) but now without the continuity and starting requirements $(2.4 \mathrm{~b})$ and (2.4c) for $Z_{N} \cup$ $\{a\}$. For each $k=0,1, \ldots, N-1,(2.4 \mathrm{a})$ yields, for a strictly monotone operator $T$, a uniquely defined $\Phi_{k}$ and hence a unique $\Phi \in S_{m-1}^{(-1)}\left(Z_{N}\right)$.

We then have (again by observing (2.8)) the following result, in analogy with Theorem 1 .

Theorem 3. Assume:

(i) The operator $T$ in (1.4) is strictly monotone.

(ii) For a fixed $k, 0 \leqslant k \leqslant N-1$, the (unique) approximation $\Phi \in S_{m-1}^{(-1)}\left(Z_{N}\right)$ to $y$ on $\left(x_{0}, x_{k+1}\right]$ has been found by (2.4a) (collocation on the sets $X_{\mu}: \mu=0$, $\ldots, k)$.

If the $\left\{\omega_{k, j}: j=1, \ldots, m\right\}$ denote the Lagrange fundamental polynomials 
for the set $Q_{k}^{\prime}$, then the values $\left\{\Phi_{k, \nu}: \nu=1, \ldots, m\right\}$ of $\Phi$ on $Q_{k}^{\prime}$ are given by

$$
\begin{aligned}
& \int_{x_{k}}^{\xi_{k, j}} K\left(\xi_{k, j}, t\right) \sum_{\nu=1}^{m} \Phi_{k, \nu} \omega_{k, \nu}(t) d t \\
& \quad=g\left(\xi_{k, j}\right)-\sum_{\mu=0}^{k-1} \int_{x_{\mu}}^{x_{\mu+1}} K\left(\xi_{k, j}, t\right) \Phi_{\mu}(t) d t, \quad j=1, \ldots, m
\end{aligned}
$$

(with the improper integrals defined in the usual manner).

As has already been indicated implicitly, the above construction of $\Phi \in S_{m-1}^{(-1)}\left(Z_{N}\right)$ does not require the value $y(a)$. Furthermore, we note the following:

(i) Suppose that $K \in C(S)$ (case (1.2a)). For a fixed $j, 1 \leqslant j \leqslant m$, let the integrals (2.6) occurring in (3.3) be evaluated by interpolatory quadrature (on $\left(x_{k}\right.$, $\left.\left.\xi_{k, j}\right]\right)$ using weights $\left\{w_{j, r}^{\prime}: r=1, \ldots, m\right\}$ and abscissas

$$
z_{j, r}^{(k)}=x_{k}+\eta_{j} \eta_{r} h, \quad r=1, \ldots, m
$$

(with $\left\{\eta_{j}\right\}$ defined in (2.3)). The integrals on the right-hand side of (3.3) are evaluated analogously (as before, we have $w_{j, r}^{\prime}=\eta_{j} w_{m, r}^{\prime}, r=1, \ldots, m$ ). The discretized lefthand side of (3.3) hence is

$$
\sum_{r=1}^{m} w_{j, r}^{\prime} K\left(\xi_{k, j}, x_{k}+\eta_{j} \eta_{r} h\right) \sum_{\nu=1}^{m} \Phi_{k, \nu} \omega_{k, \nu}\left(x_{k}+\eta_{j} \eta_{r} h\right) \quad(j=1, \ldots, m)
$$

It is now easily seen that the particular choice $\eta_{j}=u_{j}(j=1, \ldots, m)$, with $u_{m}=1$, yields the block method introduced by de Hoog and Weiss [6] (method (3.3)).

(ii) For operators $T$ with weakly singular kernels (case (1.2b)) discretization in (3.3) may be carried out along the lines of remark (iii) of Section 2. In particular, Gauss-type quadrature formulas will again be prime candidates in the approximate evaluation of the singular integrals occurring on the left-hand side of (3.3).

As in Section 2, we conclude our discussion with a remark on the special case $m=1$. We have

THEOREM 4. Let the kernel $K$ of $T$ in (1.4) satisfy the conditions of Theorem 1. Then the approximating function $\Phi \in S_{0}^{(-1)}\left(Z_{N}\right)$ to the exact solution $y$ of (1.4) is uniquely determined by (2.4a) and is given by

$$
\alpha_{k, 1}=\left\{\int_{x_{k}}^{\xi_{k, 1}} K\left(\xi_{k, 1}, t\right) d t\right\}^{-1} \cdot\left\{g\left(\xi_{k, 1}\right)-\sum_{\mu=0}^{k-1} \alpha_{\mu, 1} \int_{x_{\mu}}^{x_{\mu+1}} K\left(\xi_{k, 1}, t\right) d t\right\}
$$

$$
(k=0,1, \ldots, N-1) \text {. }
$$

As in Theorem 2, a number of discrete methods for solving (1.4) may be obtained from (3.5) by specifying the type of kernel of $T$ and the discretization procedure selected for evaluating the moment integrals in (3.5):

(i) If, in (3.5), we choose $\xi_{k, 1}=x_{k+1}$, and if $\Phi_{k}=\alpha_{k, 1}$ is regarded as an approximation to $y(x)$ at $x=\left(x_{k}+x_{k+1}\right) / 2$, then the resulting discretization is 
identical with the product midpoint method of Linz [12]; the classical midpoint method [10] is found by discretizing (3.5) via the (product) midpoint quadrature rule (stepsize $h / 2$ ).

(ii) The midpoint product method suggested by Weiss and Anderssen [15] for the numerical solution of (1.4) with weakly singular kernel (1.2b) is another discrete version of (3.5): here, collocation is again at $\xi_{k, 1}=x_{k+1}(k=0,1, \ldots, N-1)$, and the resulting moment integrals are treated as weighted integrals with weight functions $w_{\mu}(t)=\left(\xi_{k, 1}-t\right)^{-\lambda}(\mu=0,1, \ldots, k)$; they are then discretized by applying the (weighted) midpoint quadrature rule for each interval $\sigma_{\mu}^{\prime}$. Again, these special discretization procedures will only be suitable for smooth kernels; in general it will be advantageous to apply some efficient automatic quadrature routine [13] to the integrals in (3.5).

4. Concluding Remarks. If the Volterra operator $T$ in (1.4) is strictly monotone, i.e. if $T$ satisfies one of the corresponding pairs of conditions in (1.2) and (1.3), and if the integrals in (2.4a) (left-hand side) are computed exactly, then the resulting matrix $C_{k}$ in (2.5) will be nonsingular for any choice of $X_{k}$ and $Q_{k}(k=0,1, \ldots$, $N-1$ ). (We note in passing that condition (1.3) may be weakened: in (1.3a), for example, $K$ is allowed to vanish at some (or all) points $x=t \in I$, i.e. $K(x, x)=0, x$ $\in I$, with $|K(x, t)|>0, a \leqslant t<x \leqslant b$.) By continuity, this will remain true if the discretization procedure applied to $C_{k}$ is such that the integrals (2.6a) are approximated to sufficient accuracy (by making use of an appropriate automatic quadrature routine).

However, for $m>1$, and for a fixed $h>0$, this will no longer hold in general (again by continuity arguments) if $C_{k}$ is discretized by a given fixed discretization procedure which does not take into account the nature of the kernel of $T$. In the case $m=1$ the situation is different: strict monotonicity of $T$ implies that the discretized equations (2.11) and (3.5), respectively, possess unique solutions provided the quadrature formula used has positive weights (as in Gauss quadrature, for example). Also, if discretization is based on the (simple) trapezoidal rule (for (2.11)), or the (simple) midpoint rule (for (3.5)), then strict monotonicity of $T$ is no longer required for the existence of a unique solution: the corresponding discrete regularity conditions reduce to $K(x, x) \neq 0, x \in Z_{N} \cup\{b\}$ and $K(x, x-h / 2) \neq 0, x \in Z_{N} \cup\{b\}$, respectively.

Finally, it is trivial that the above discussion applies to (regular or weakly singular) Volterra integral equations of the second kind,

$$
y(x)+(T y)(x)=g(x), \quad x \in I,
$$

with $T$ given by (1.1). The product-type method of [11] and the Runge-Kutta-type methods of [8] again correspond to particular choices of the sets $\left\{X_{k}\right\}$ and the discretization procedure for the resulting moment integrals. Here again, a distinction between regular and weakly singular kernels has only to be made when selecting the discretization method, and it will in general also be appropriate to base it on some efficient automatic quadrature routine. 
Department of Mathematics

Dalhousie University

Halifax, Nova Scotia, Canada

1. C. T. H. BAKER, "Methods for Volterra equations of the first kind," Numerical Solution of Integral Equations (L. M. Delves \& J. Walsh, Editors), Clarendon Press, Oxford, 1974, pp. 162174.

2. W. R. BOLAND \& C. S. DURIS, "Product type quadrature formulas," BIT, v. 11, 1971, pp. 139-158. MR 45 \#1382.

3. H. BRUNNER, "The solution of nonlinear Volterra integral equations by piecewise polynomials," Proc. Manitoba Conf. on Numerical Mathematics (R. S. D. Thomas \& H. C. Williams, Editors), Univ. of Manitoba, Winnipeg, Canada, 1971, pp. 65-78. MR 49 \#1812.

4. H. BRUNNER, "Global solution of the generalized Abel integral equation by implicit interpolation,” Math. Comp., v. 28, 1974, pp. 61-67. MR 48 \#10162.

5. H. BRUNNER, "On the approximate solution of first-kind integral equations of Volterra type," Computing, v. 13, 1974, pp. 67-79.

6. F. de HOOG \& R. WEISS, "On the solution of Volterra integral equations of the first kind," Numer. Math., v. 21, 1973, pp. 22-32. MR 51 \#7335.

7. F. de HOOG \& R. WEISS, "High order methods for Volterra integral equations of the first kind," SIAM J. Numer. Anal., v. 10, 1973, pp. 647-664. MR 51 \#9554.

8. F. de HOOG \& R. WEISS, "Implicit Runge-Kutta methods for second kind Volterra integral equations," Numer. Math., v. 23, 1974/75, pp. 199-213. MR 51 \#9549.

9. H. S. HUNG, The Numerical Solution of Differential and Integral Equations by Spline Functions, MRC Technical Report \#1053, Math. Res. Center, Univ. of Wisconsin, Madison, 1970.

10. P. LINZ, "Numerical methods for Volterra integral equations of the first kind," Comput. J., v. 12, 1969, pp. 393-397. MR 40 \#6791.

11. P. LINZ, "Numerical methods for Volterra integral equations with singular kernels," SIAM J. Numer. Anal., v. 6, 1969, pp. 365-374. MR 41 \#4850.

12. P. LINZ, "Product integration methods for Volterra integral equations," $B I T$, v. 11 , 1971, pp. 413-421. MR 47 \#2848.

13. J. N. LYNESS \& J. J. KAGANOVE, EAR Report QR2 (May 1975 version), Argonne National Laboratory, Argonne, IIl., 1975.

14. R. WEISS, "Product integration for the generalized Abel equation," Math. Comp., v. 26, 1972, pp. 177-190. MR 45 \#8050.

15. R. WEISS \& R. S. ANDERSSEN, "A product integration method for a class of singular first kind Volterra equations," Numer. Math., v. 18, 1971/72, pp. 442-456. MR 47 \#1314.

16. A. YOUNG, "The application of approximate product-integration to the numerical solution of integral equations," Proc. Roy. Soc. London Ser. A, v. 224, 1954, pp. 561-573. MR $16,179$. 